\title{
Determination of carrageenan in jellies with new methylene blue dye using spectrophotometry, smartphone-based colorimetry and spectrophotometric titration
}

\author{
Marie BARTLOVÁ ${ }^{1}$ (D), Dorota ZIÓŁKOWSKA ${ }^{2}$ (D), Matej POSPIECH ${ }^{1 \star}$ (D), Alexander SHYICHUK ${ }^{2}$ (D), \\ Bohuslava TREMLOVÁ ${ }^{1}$ (ic)
}

\begin{abstract}
Carrageenan is a seaweed polysaccharide widely used as a food gelling and thickening agent. The New Methylene Blue dye was tested as an analytical reagent for the quantification of carrageenan in commercial jelly samples. The main spectral peaks of the dye decrease with increasing carrageenan-to-dye molar ratio up to 1 . Three analytical procedures were tested: (i) spectrophotometric measurement at $590 \mathrm{~nm}$, (ii) smartphone-based color measurement within the RGB color model and (iii) spectrophotometric titration at $625 \mathrm{~nm}$. All the procedures provide straight calibration lines. Spectrophotometry provides better linearity as compared to smartphone-based colorimetry. Range of determination is dependent on type and purity of carrageenan. The spectrophotometric titration procedure provides lower values of limit of determination due to longer optical path used. Typical food additives used in jelly deserts do not interfere significantly with the proposed analytical approach.
\end{abstract}

Keywords: spectrophotometric titration; phenothiazine dye; New Methylene Blue N dye; sulfated polysaccharides; carrageenan.

Practical Application: Development of the methods for carrageenan detection.

\section{Introduction}

Carrageenan is a seaweed-derived sulfated polysaccharide known as food additive E407. It is used as gelling and thickening agent in cheeses, puddings, desserts and cured meats (Bednářová et al., 2014; Błaszak et al., 2018). Carrageenan macromolecules consist of $\alpha-(1-3)$-D-galactose units and $\beta$-(1-4)-3,6-anhydro-D-galactose units. They may differ in the content and position of sulfate groups. The most common food-grade varieties are kappa $(\kappa)$ and iota $(l)$ carrageenans used as gelling, stabilizing and emulsifying agents, as well as lambda $(\lambda)$ carrageenan used as a thickener. The market demand for carrageenan is constantly growing (Campbell \& Hotchkiss, 2017). Carrageenan is generally recognised as safe, however, products of its hydrolysis can cause adverse effects on human health (McKim et al., 2018).

Analytical tasks of carrageenan quantification concern mainly food and cosmetics products. Detection of carrageenan in meat samples can be carried out by means of histochemical method using combined staining by Periodic Acid Schiff and Calleja stains (Bednářová et al., 2014; Petrášová et al., 2016). The very sensitive and accurate HPLC technique requires previous separation of carrageenan from the food matrix. All kinds of proteins bind strongly to carrageenan due to interaction with anionic groups (Blakemore et al., 2014; Weiner 2016). That is why the HPLC procedure includes methanolysis followed by quantification of 3,6-anhydrogalactose dimethylacetal (Quemener et al., 2000). The cited methods do not distinguish between food grade carrageenan ( $\mathrm{Mw} 200$ to $800 \mathrm{kDa}$ ), low molecular weight polygeenan (Mw $10-20 \mathrm{kDa}$ ) and hydrolyzed carrageenan (Mw 20-40 kDa) (McKim et al., 2018). The low-molecular-weight fractions of carrageenan can be detected with LC-MS technique (Blakemore et al., 2014).

Industrial laboratories routinely assess the amount of carrageenan in both raw materials and final products. For this purpose, a simplified analytical procedure based on determination of sulphate groups seems to be sufficient. The very convenient method of determination of anionic groups regardless of carrageenan molecular weight is spectrophotometry with the Methylene Blue (MB) dye (Soedjak 1994). The MB dye form ion associates with anionic polymers resulting in decrease and shift of the main absorbance peak (Coronel et al., 2017; Stîngă et al., 2019). The spectral maximum undergoes hypsochromic shift from $664 \mathrm{~nm}$ (blue color) to $559 \mathrm{~nm}$ (purple color) (Herfurth \& Ulrich, 2017). Absorbance at $664 \mathrm{~nm}$ corresponds to monomer form of the dye while absorbance at $559 \mathrm{~nm}$ corresponds to dimer form. The dimers and higher aggregates of the MB dye have sandwich-type structure (Lakshminarasimhan et al., 2017). Another phenothiazine dye, Toluidine Blue (TB), has structure similar to the MB dye. The structural difference is position and quantity of methyl groups in the dye molecules (Figure Aa, Ab in Supplementary material). The TB dye reveals metachromatic properties very similar to those of the MB dye. Spectral maximum of the TB dye associated with anionic polymer is shifted from 640 to $540 \mathrm{~nm}$ (Ziółkowska et al., 2017). Both the dyes have been used for quantitation of carrageenan in a

${ }^{1}$ Faculty of Veterinary Hygiene and Ecology, University of Veterinary and Pharmaceutical Sciences Brno, Palackeho, Brno, Czech Republic

${ }^{2}$ Faculty of Chemical Technology and Engineering, UTP University of Science and Technology, Seminaryjna, Bydgoszcz, Poland

*Corresponding author: mpospiech@vfu.cz 
photometric manner. For example, the MB dye was applied for determination of carrageenan solubility (Serowik et al., 2018). The TB dye enabled determination of carrageenan binding to casein and cell proteins (Sokolova et al., 2017). Both the $\mathrm{MB}$ and $\mathrm{TB}$ dyes proved to be applicable in automatic photometric titration procedure (Ziółkowska et al., 2017, 2018). This work concerns application of another phenothiazine dye, New Methylene Blue N (NMB), for the determination of carrageenans of different types. Compared to the TB and MB dyes, the NMB dye molecule possesses two methyl groups attached directly to the aromatic structure (Figure A in Supplementary material). The dye structure is favourable to form stacked aggregates with polyanions. For example, affinity of the NMB dye to polyadenylic acid is quite strong and comparable to that of the TB dye (Paul et al., 2017). The obvious structural similarity of both the dyes suggests the NMB dye to be a suitable reagent for the quantification of carrageenan.

\section{Materials and methods}

\subsection{Materials and reagents}

Reagent-grade kappa carrageenan and New Methylene Blue $\mathrm{N}$ dye $\left(\mathrm{C}_{18} \mathrm{H}_{22} \mathrm{ClN}_{3}\right.$ S, CAS 1934-16-3, C.I. 52030) came from Sigma Aldrich. Technical-grade carrageenan samples approved according to EU regulation 2012/231/EU were the following: refined kappa and iota carrageenans E407 from Eurogum A/S (Eurogum, Denmark) as well as refined iota carrageenan E407 and semi-refined iota carrageenan E407a (guma euchema) from Kerry Ingredients \& Flavours (Kerry, Ireland). Citrus pectin WEC-6 was obtained from Pektowin (Poland), potassium sodium tartrate and potassium phosphate came from POCh (Poland). Glucose, apple juice NFC 100\%, cola aroma and pineapple aroma came from local market. The real food samples were three jellies, i.e. colorless cake jelly (Dr.Oetker), colorless and red dessert jellies (Delecta), as well as energy gel cola (Concap). Their compositions are presented in Table 1.
Spectrophotometric and smartphone-based colorimetric measurements

Samples for the spectrophotometric and colorimetric measurements were prepared by mixing $1 \mathrm{~mL}$ of carrageenan solution (variable concentration, from 5 to $400 \mathrm{mgL}^{-1}$ ) with $5 \mathrm{~mL}$ of NMB solution (constant concentration, $50 \mu \mathrm{M}$ ). Spectra of the obtained solutions were measured using Spectroquant ${ }^{\mathbb{B}}$ Pharo 300 spectrophotometer (Merck, Germany). Spectra were recorded using quartz cuvettes and glass test tubes with optical paths of $10 \mathrm{~mm}$ and $14 \mathrm{~mm}$, respectively. Color images of solutions in test tubes were taken with a smartphone (Samsung Galaxy S3 mini) against flat LED lamp (11 klux, $3500 \mathrm{~K}$ ). The light source was placed closely behind the test tube (Figure D in Supplementary material). Numerical values of the RGB additive color model were taken from the mid of the images using HTML Color Picker (2020). Samples for interference tests consisted of $0.2 \mu$ mole of NMB, $0.06 \mathrm{mg}$ of refined iota carrageenan Kerry, varying quantities of examined food additive and water up to $4 \mathrm{~mL}$. The jellies from Dr.Oetker and Delecta (which have a form of dry powder) were pre-dissolved in hot water and cooled to room temperature. Then all the food samples were diluted with distilled water to a concentration corresponding to the range of the standard curve.

\section{Spectrophotometric titration}

Spectrophotometric titration graphs were obtained at wavelength $625 \mathrm{~nm}$ using Spekol 11 spectrophotometer (Carl Zeiss, Germany) equipped with magnetically stirred titration cuvette of $2 \mathrm{~cm}$ optical path and $30 \mathrm{~mL}$ in volume. The $\mathrm{NMB}$ dye solution with concentration $1 \mathrm{mM}$ was added stepwise, with $10 \mu \mathrm{L}$ increment, to kappa carrageenan standard solution of volume $20 \mathrm{~mL}$ using electronic burette OP-930/1 (Radelkis Hungary). The variance values were calculated using numerical data of tenfold determination of the same carrageenan in solution of $30 \mathrm{mgL}^{-1}$. Real food samples were prepared as described previously.

Table 1. Components of the jellies studied.

\begin{tabular}{ccccc}
\hline component & colorless cake jelly Dr.Oetker & colorless dessert jelly Delecta & red dessert jelly Delecta & energey gel cola Concap \\
\hline glucose & & & \\
fructose & + & + & + \\
sucrose & & + & + \\
pectin & + & + & + \\
maltodextrin & + & + & + \\
carrageenan & & + & + \\
locust bean gum & & + & + \\
citric acid & + & + & + \\
potassium sorbate & & + & + \\
potassium sodium tartrate \\
potassium tartrate (plural \\
form)
\end{tabular}




\subsection{Statistical analysis}

Statistical analysis was carried out by means of Unistat 6.0 software (Unistat ltd, GB) using Kruskal-Wallis test for method comparison.

\section{Results and discussion}

Three methods for the quantification of carrageenan were tested. All the proposed procedures are based on spectral changes of the NMB dye. The UV-Vis absorption spectrum of the NMB dye has four peaks: two of them are situated in the ultraviolet range (Figure 1a) and the next two peaks are situated in the visible range (Figure $1 \mathrm{~b}$ ). The spectral peaks at $623 \mathrm{~nm}$ and $588 \mathrm{~nm}$ (Figure 1b) are attributed to the monomer and dimer forms of the NMB dye, respectively. The NMB dye spectrum is a little bit shifted to shorter wavelengths as compared to the TB dye.

Addition of carrageenan leads to substantial changes of the NMB dye spectrum. The main spectral peaks are decreased with the increase of carrageenan amount up to $100 \mathrm{mgL}^{-1}$. (Figures $1 \mathrm{a}, 1 \mathrm{~b}$ ).
Absorbance changes in ultraviolet range (Figure 1a) are similar to those observed in visible range. Further increase of carrageenan amount (up to $200 \mathrm{mgL}^{-1}$ ) results in formation of a new broad band with a maximum situated at $547 \mathrm{~nm}$ (Figure 1b). The registered spectral changes are characteristic for other phenothiazine dyes, too. Suchlike spectral changes are usually ascribed to formation of ion associates (Soedjak 1994; Coronel et al., 2017; Paul et al., 2017; Stîngă et al., 2019).

In contrast with the UV-vis range, absorbance in the nearest infrared range increases slowly with the carrageenan amount (Figure 1c). The flat falling spectra are characteristic for light scattering on fine particles. Hence, the spectral changes presented in Figure 1c suggest formation of a dilute suspension. The probable cause is low solubility of carrageenan-dye ion associates.

The spectra presented in Figures 1a and $1 \mathrm{~b}$ contain so called isosbestic points at 323, 545 and $679 \mathrm{~nm}$, marked by red circles. At these characteristic wavelengths, the absorbance values remain constant and do not depend on the concentration of carrageenan
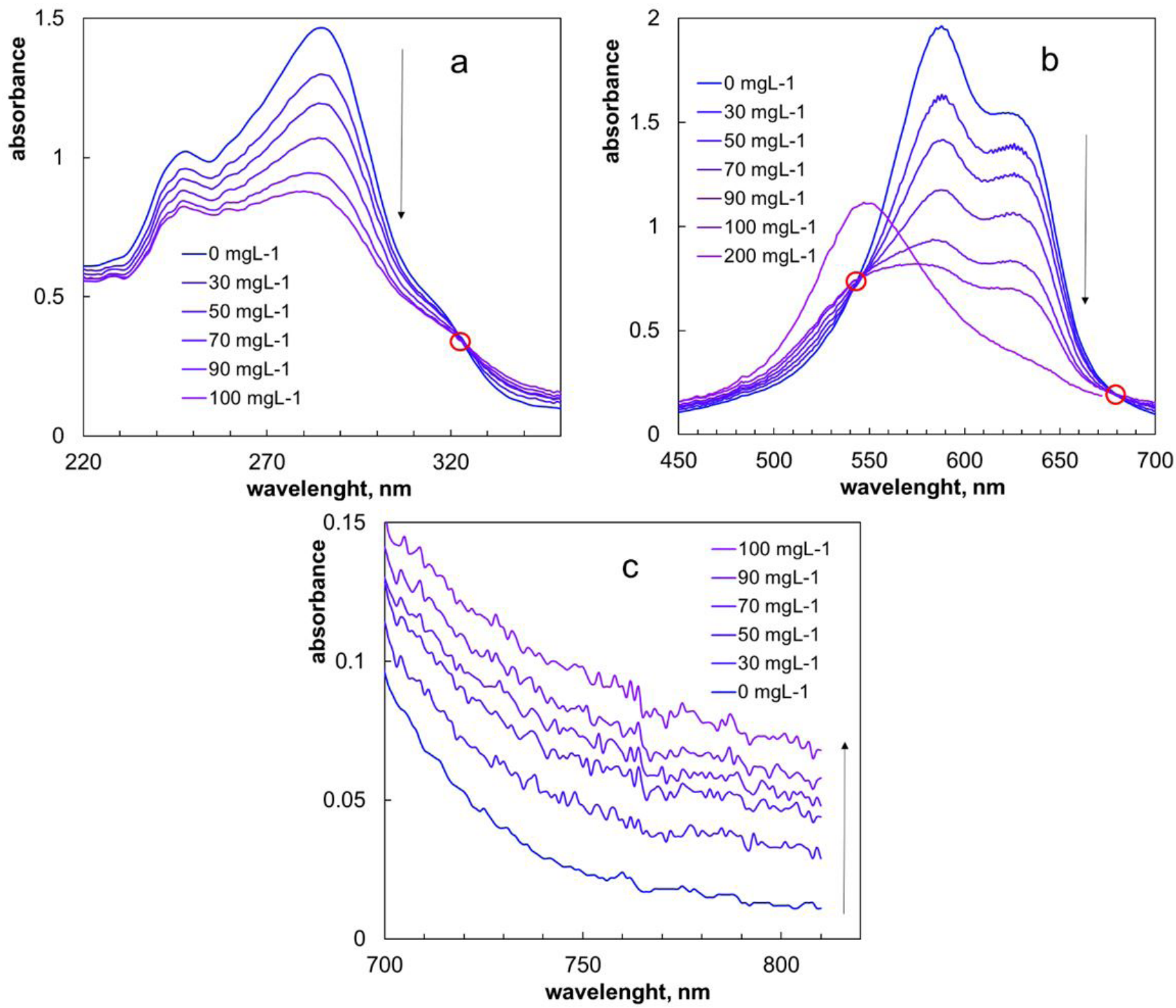

Figure 1. UV-vis spectra of reaction mixtures. Kappa Sigma carrageenan concentrations are indicated. The optical path is $10 \mathrm{~mm}$. 
(Figure B in Supplementary material). Presence of isosbestic points suggests that the ion associates have stoichiometric nature. The isosbestic points are observed until the molar amount of carrageenan sulphate groups is less than amount of the dye (Figure B in Supplementary material). The isosbestic points disappear when the molar ratio of sulphate groups to dye molecules exceeds 1:1 (Figure 1b). One can conclude that the NMB dye forms stoichiometric associates with carrageenan at polymer-to-dye ratio below 1 . The broad band at $547 \mathrm{~nm}$ is also formed when the polymer-to-dye molar ratio exceeds 1 (Figure 1b). Hence, the band at $547 \mathrm{~nm}$ can be attributed to non-stoichiometric supramolecular agglomerates of carrageenan and the NMB dye.

Spectral changes induced by commercial carrageenan samples (Figures $\mathrm{Ca}$ - Cc in Supplementary material) are very similar to the ones induced by the reagent-grade kappa carrageenan from Sigma (Figure 1b). In all the cases, addition of carrageenan leads to decrease of the main peaks in the dye spectrum. However, the amount of carrageenan needed to change the spectrum shape is dependent on carrageenan type and sample purity (Figure C in Supplementary material). The first explanation of the impact of carrageenan type (compare Figures $\mathrm{Ca}$ and $\mathrm{Cb}$ in Supplementary material) is different content of sulphate groups in the samples studied. Iota carrageenan macromolecules bear two sulphate groups per disaccharide unit whereas kappa carrageenan macromolecules bear one sulphate group per disaccharide unit. However, the data in Figure C in Supplementary material show rather a small difference between kappa and iota carrageenans obtained from the same manufacturer. Hence, the second probable explanation of the difference between spectra recorded for carrageenan kappa and iota samples is presence of unknown admixtures. In reality, food-grade carrageenan may contain admixtures of potassium chloride, non-sulphated polysaccharides, sucrose etc. Furthermore, effect of the carrageenan sample purity on the observed phenomenon may be more significant than effect of the carrageenan type. Comparing Figures $\mathrm{Cb}$ and $\mathrm{Cc}$ in Supplementary material, one can notice, that refined carrageenan is twofold more active towards the dye as compared to semi refined carrageenan.

Spectra of the carrageenan-dye mixtures proved to undergo slow changes in time. Figure 2 illustrates decrease of absorbance at chosen wavelengths, drawn for samples with low and medium concentration of the carrageenans discussed. The probable cause is formation of a fine suspension of the ion associates. Increased absorbance at long wavelengths beyond the main spectral peak confirms presence of suspended particles (Figure 1c). Similarly, both the $\mathrm{MB}$ and $\mathrm{TB}$ dyes are known to form fine suspensions with carrageenan (Herfurth \& Ulrich 2017; Ziółkowska et al., 2017). The absorbance changes in time are dependent on both carrageenan type and concentration (Figures 2). The kinetic experiments have shown that the absorbance changes are more rapid in the case when the concentration of carrageenan is low (compare Figures 2a and 2b). At large carrageenan concentrations, i.e. far above equimolar ratio, micrometer-sized agglomerates may form. Similar phenomenon was observed in mixtures of carrageenan with other phenothiazine dyes (Ziółkowska et al., 2017 ) and mixtures of cationic polymers with anionic dyes (Ziółkowska et al., 2014). Fortunately, formation of large aggregates occurs rather slowly. Because the spectra change over time, analytical measurements should be performed within a short time after adding the NMB dye reagent. Time window 10-15 min after reagent adding seems to be sufficient to perform spectral measurements and provide accurate results.

Spectral changes of the NMB dye (Figures 1 and Figures C in Supplementary material) are the basis of the proposed analytical procedures. Absorbance values plotted vs. carrageenan concentration resulted in straight lines well suitable for the analytical purpose (Figure 3a). The calibration graphs have similar slopes at wavelengths in the Vis range from 590 to $630 \mathrm{~nm}$ (Table 2). However, the calibration graph in the UV range (at $285 \mathrm{~nm}$ ) has decreased slope (Figure 3a, Table 2). The common disadvantage of the obtained calibration graphs

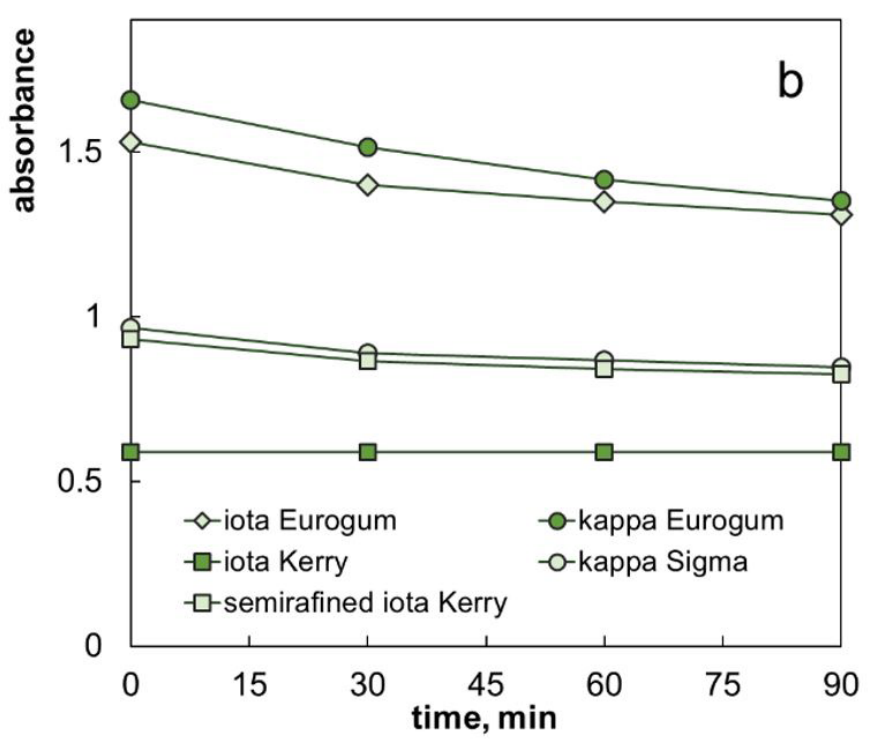

Figure 2. Changes of absorbance at $590 \mathrm{~nm}$ over time. Carrageenan concentration is $10 \mathrm{mgL}^{-1}$ (a) and $80 \mathrm{mgL}^{-1}$ (b). The optical path is $14 \mathrm{~mm}$ 

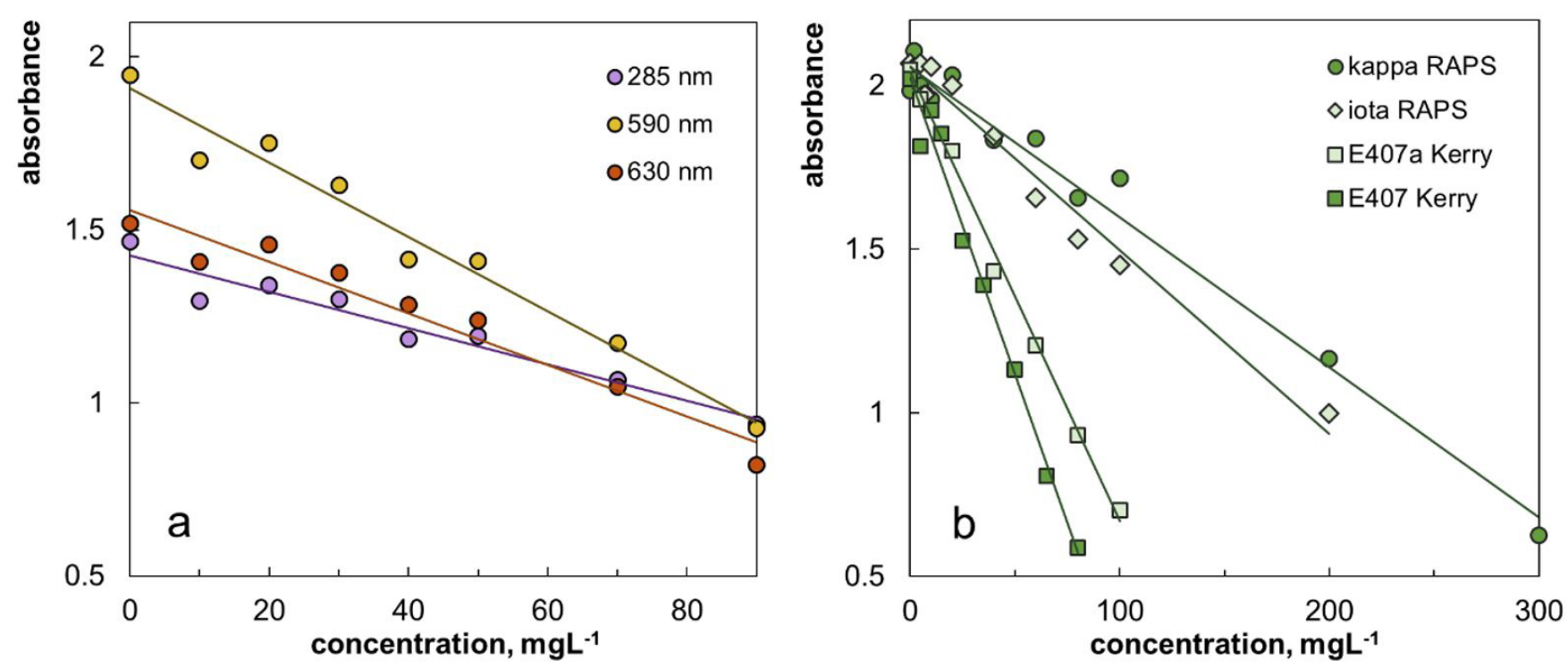

Figure 3. Calibration lines obtained by spectrophotometry: for kappa Sigma carrageenan at indicated wavelengths (a); for different carrageenans at $590 \mathrm{~nm}$ (b). The optical path is $14 \mathrm{~mm}$.

Table 2. Numerical parameters of the calibration lines obtained.

\begin{tabular}{cccccc}
\hline sample & wavelength, $\mathrm{nm}$ & optical path, $\mathrm{mm}$ & slope, $\mathrm{mLmg}^{-1}$ & $\mathrm{R}^{2}$ & range, $\mathrm{mgL}^{-1}$ \\
\hline kappa Sigma & 285 & 10 & -5.9 & 0.989 & $7-100$ \\
kappa Sigma & 630 & 10 & -8.6 & 0.969 & $7-100$ \\
kappa Sigma & 590 & 10 & -11.5 & 0.992 & $7-100$ \\
kappa Eurogum & 590 & 14 & -5.1 & 0.941 & $20-300$ \\
iota Eurogum & 590 & 14 & -6.5 & 0.982 & $20-200$ \\
semi-refined iota Kerry & 590 & 14 & -13.6 & 0.989 & 0.982 \\
iota refined Kerry & 590 & 14 & -17.9 & & $20-80$ \\
\hline
\end{tabular}

Table 3. Tolerance ratio towards carrageenan.

\begin{tabular}{cc}
\hline additive & ratio, w/w \\
\hline locust bean gum & 100 \\
pectin & 1.5 \\
sucrose & 50 \\
glucose & $>160$ \\
citric acid & 0.8 \\
potassium sodium tartrate & $<0.3$ \\
potassium phosphate & $<0.3$ \\
concentrated fruit (apple) juice & $0.2^{\star}$ \\
cola aroma oil & $0.05^{\star}$ \\
pineapple aroma oil & $0.3^{\star}$ \\
\hline
\end{tabular}

*v/w $[\mathrm{mL} / \mathrm{g}]$.

is low repeatability at small concentrations of carrageenan. Therefore, the lower limit of carrageenan determination was estimated to be $7 \mathrm{mgL}^{-1}$. On the other hand, the upper limit of determination is somewhat higher as compared to the one obtained with the TB dye (Ziółkowska et al., 2017).

Technical-grade carrageenan samples also resulted in straight calibration lines (Figure 3b). The calibration lines proved to have quite different slopes (Figure $3 \mathrm{~b}$, Table 2). The slopes decrease in the following order: kappa (Eurogum) - iota (Eurogum) semi-refined iota (Kerry) - iota (Kerry). In full accordance, the ranges of determination decrease in the same order (Table 2). The observed difference between kappa (Eurogum) and iota (Eurogum) carrageenan samples may be explained by different average numbers of sulphate groups per structural unit, as described earlier. The difference between iota refined (Kerry) and iota semi-refined (Kerry) carrageenan samples is evidently related to the content of plant fibers. In turn, the differences between carrageenan iota samples from different manufacturers may be due to the presence of hybrid type carrageenans (Campbell \& Hotchkiss 2017). Commercial samples of food-grade carrageenan may also contain admixtures of sucrose and dextrose (Herfurth \& Ulrich 2017).

In general, food additives other than carrageenan may disturb the calibration lines. For that reason, a series of food additives were tested for interference exclusion. The effect of some additives typical for jelly desserts have been investigated earlier (Ziółkowska et al., 2017). In this work, next additives typical for jelly deserts were tested. All the tested additives showed no significant interference. In most cases, typical quantities of additives present in jellies are less than the values shown as a limit of tolerance in Table 3.

The above described spectral changes of carrageenan-NMB mixtures are accompanied with well-visible color changes. Figure $\mathrm{E}$ in the Supplementary material shows a series of images taken with a smartphone from calibration solutions. With the increase of 


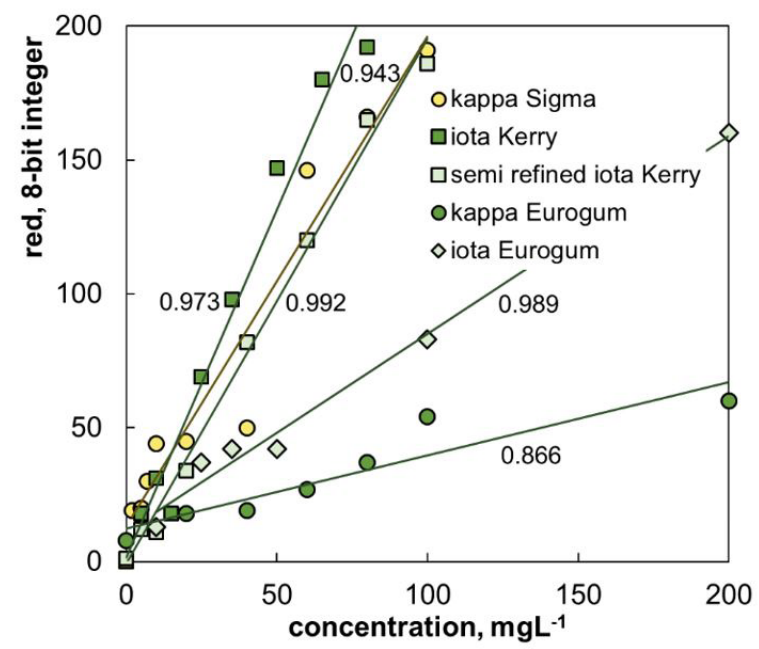

Figure 4. Correlation between Red color component and absorbance at $590 \mathrm{~nm}$.

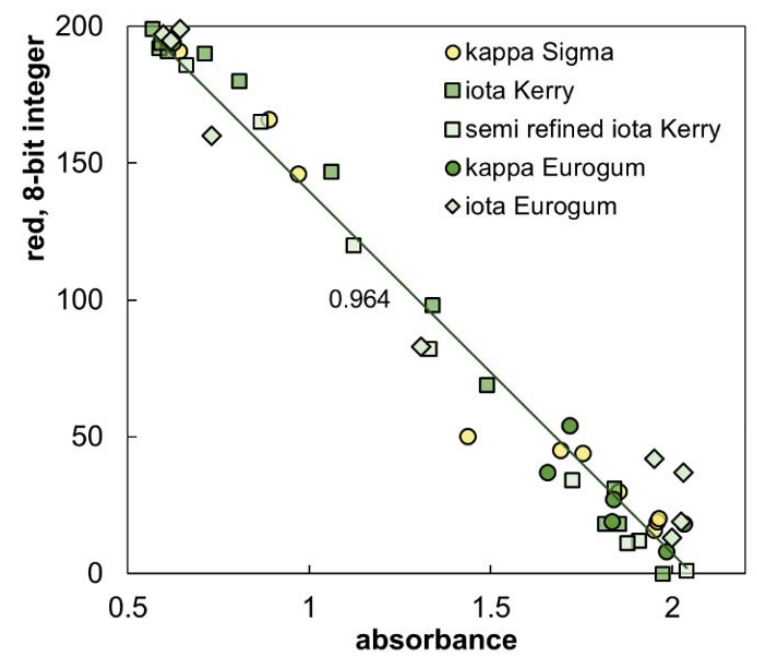

Figure 5. Calibration lines based on Red color component.

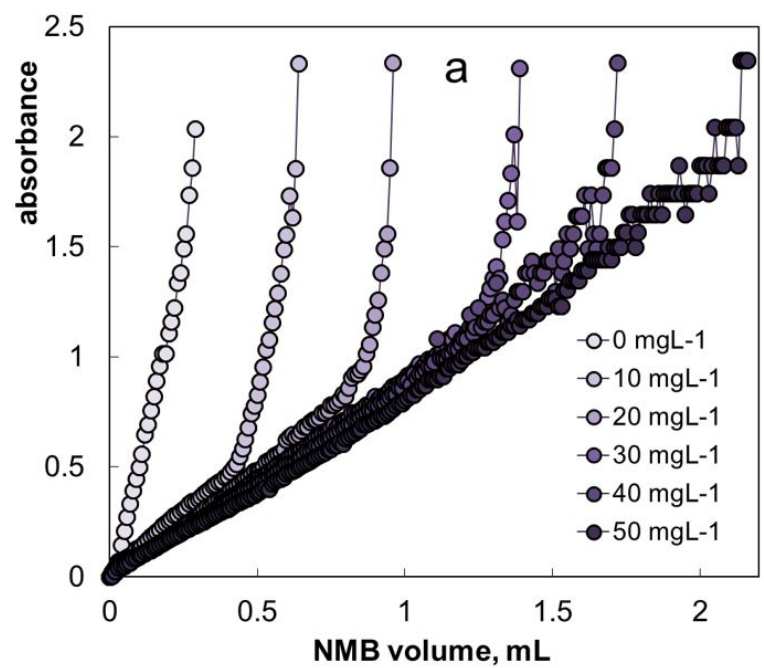

carrageenan amount, color of the solutions is changed from blue to pink (Figure E in Supplementary material). It is well known that numerical values of color can be used for analytical purposes (Kılıç et al., 2018; Rateni et al., 2017; Porto et al., 2019). Using the obtained color images, a simplified colorimetric calibration was performed as well. Among numerical values of the RGB additive color model, the Red one provides the most sensitive analytical signal. Relations between the values of Red color component and absorbance at $590 \mathrm{~nm}$ proved to be the same for all the carrageenan samples studied (Figure 4). This fact indicates a good agreement between smartphone-based colorimetry and standard spectrophotometry data. The calibration graphs obtained via red color photometry proved slightly less accurate as compared to the ones plotted using absorbance values (compare numerical values of determination coefficients, $\mathrm{r}^{2}$, presented in Figure 5 and Table 2). The $r^{2}$ values resulted from smartphone-based method were worse (for kappa (Eurogum), iota (Kerry), kappa (Sigma)) or comparable (for iota (Eurogum), iota semi refined (Kerry)) to those resulted from the spectrophotometric method. However, the RGB color measurements can find wider applicability due to widespread use of smartphones.

Spectrophotometric titration technique was also tested using the reagent-grade carrageenan (Sigma) as a model analyte. The obtained results are similar to the ones obtained with the Methylene Blue and Toluidine Blue dyes (Ziółkowska et al., 2017). The spectrophotometric titration graphs contain two parts with different slopes (Figure 6a). The first part with low slope corresponds to formation of carrageenan-dye ion pairs. The second part with larger slope corresponds to increase of free dye amount. Appearance of free dye in the solution is a gradual process. As a result, the obtained spectrophotometric graphs do not contain clear inflection points indicating the equimolar end point. Therefore, the titration is carried out using relative end points. The concept of treatment of photometric titration curves without a clearly recognizable endpoint was described elsewhere (Ziółkowska et al., 2017). Titration is carried out until a predefined absorbance value (e.g. $0.5,1,1.5,2$ or other) is

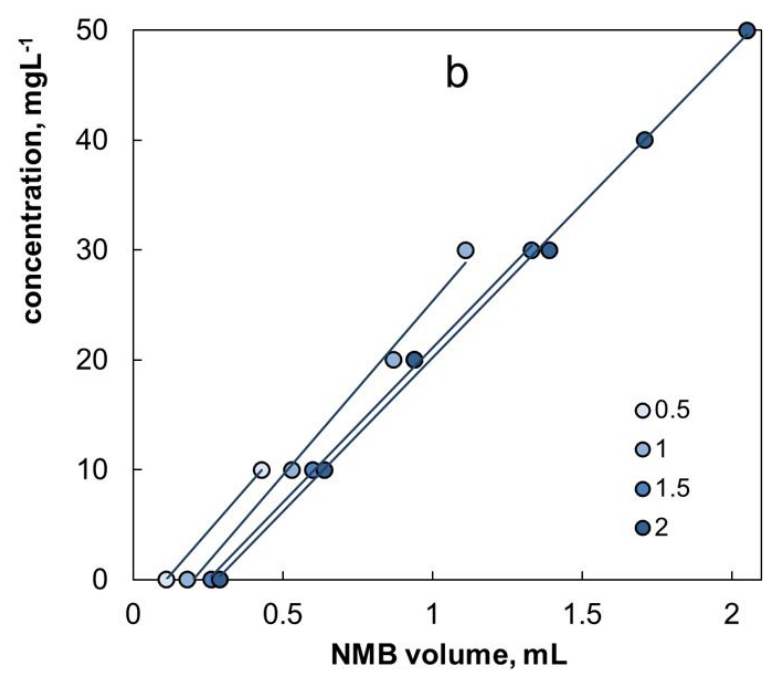

Figure 6. Photometric titration curves at indicated concentrations of kappa Sigma carrageenan (a); calibration lines resulting from spectrophotometric titration (b). 
reached. The volume of titrant required to reach this absorbance value is used as the titration end-point. The calibration curves are dependences between analyte amount and volume of titrant used for reaching the predefined absorbance value. In this way, several calibration graphs may be determined. Usually, careful selection of the end-point absorbance is needed to obtain good results.

The appropriate values of end point absorbance for the carrageenan-NMB system lie in the range 1-2.4 (Figure 6b). The larger is the end point absorbance, the broader is the determination range (Figure $6 \mathrm{~b}$ ). The proposed titration procedure

Table 4. Coefficient of determination of calibration graphs, determination range and relative standard deviation (RSD) of the titration procedure.

\begin{tabular}{cccc}
\hline absorbance & $\mathrm{R}^{2}$ & range, $\mathrm{mgL}^{-1}$ & $\mathrm{RSD}, \%$ \\
\hline 0.5 & 1.000 & $0-10$ & 3.12 \\
1 & 0.993 & $0-30$ & 3.83 \\
1.5 & 0.999 & $0-30$ & 2.21 \\
2 & 0.998 & $0-50$ & 2.63 \\
\hline
\end{tabular}

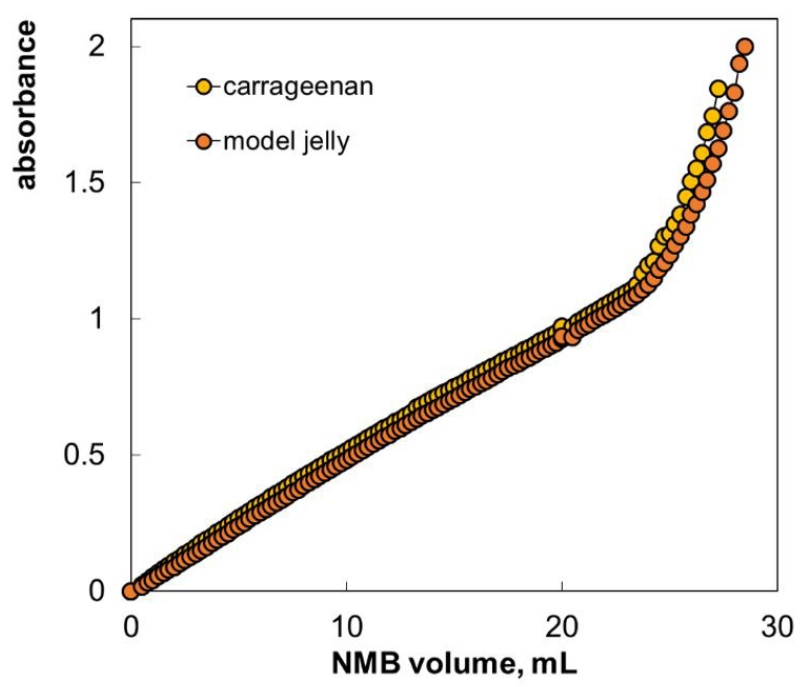

Figure 7. Titration curves obtained for carrageenan solution and model jelly solution. Carrageenan dose is $7 \mathrm{gL}^{-1}$.

Table 5. Model jelly composition.

\begin{tabular}{cc}
\hline component & g per $500 \mathrm{~mL}$ \\
\hline sucrose & 70 \\
kappa carrageenan & 3.5 \\
citric acid & 0.8 \\
sodium citrate & 0.7 \\
Brilliant Blue FCF & 0.01 \\
\hline
\end{tabular}

ensures good accuracy of carrageenan determination. The best results can be obtained with the end point absorbance equal to 1.5 (Table 4). The limits of determination with the NMB dye proved to be comparable with the ones obtained with the TB dye (Table 4, (Ziółkowska et al., 2017)).

Influence of additives on results of titrimetric method was determined as well. Titration curves obtained for carrageenan solution and model jelly solution containing the same dose of carrageenan (Figure 7) are almost the same. The observed difference does not exceed 3\%. The components of the model jelly are listed in Table 5 .

The new methods were used to determine carrageenan in commercial samples: three jellies and energy gel. The obtained results are presented in Table 6 . In general, the obtained values of carrageenan content are consistent with the texture of the products tested. The Dr. Oetker jelly is to form a hard crust that will be able to hold fruits on the surface of a cake. For this reason, this jelly has the highest carrageenan content among the products tested. The Delecta jellies are intended for making soft sweet deserts. Therefore, the carrageenan content is one third of that in the hard jelly (Table 6). In turn, the Concap energy gel is a viscous liquid intended for drinking. That is why this product has the lowest carrageenan content.

However, the results of carrageenan determination obtained for a given product with the three methods show some differences (Table 6). While smartphone-based colorimetry and spectrophotometry provided rather similar numerical data, the results of spectrophotometric titration are quite different. For the hard jellies, the determined carrageenan contents increase in the order: spectrophotometric titration < spectrophotometry $<$ smartphone-based colorimetry. For the Concap energy gel, the determined carrageenan contents increase in the order: spectrophotometry < smartphone-based colorimetry $<$ spectrophotometric titration. For the products with high carrageenan content, significant differences were recorded between the results of smartphone-based colorimetry and photometric titration $(\mathrm{A}=1 ; 1.5 ; 2)(\mathrm{p}<0.05)$. Likewise, significant differences were observed between the results of spectrophotometry and spectrophotometric titration $(A=1.5)(\mathrm{p}<0.05)$. For the product with low carrageenan contents, the differences between the results were less pronounced (Table 6). Again, significant differences were observed between the numerical results of smartphone-based colorimetry, spectrophotometry and photometric titration $(\mathrm{A}=1 ; 1.5 ; 2)(\mathrm{p}<0.05)$.

The values of Relative Standard Deviation (Table 7) show that results of all the three methods are well repeatable. The lowest RSD values were registered for smartphone-based

Table 6. Carrageenan content (g/100 g) in commercial jellies determined with different methods.

\begin{tabular}{cccrcrc}
\hline \multirow{2}{*}{ sample } & \multirow{2}{*}{ red color photometry } & \multirow{2}{*}{ spectrophoto-metry } & \multicolumn{3}{c}{ spectrophotometric titration at predefined absorbance } \\
\cline { 4 - 7 } & & & 0.5 & 1 & 1.5 & 2 \\
\hline colorless cake jelly Dr.Oetker & $12.88^{\mathrm{c}}$ & $11.66^{\mathrm{bc}}$ & 10.08 & $10.48^{\mathrm{a}}$ & $10.95^{\mathrm{ab}}$ & $10.24^{\mathrm{a}}$ \\
red dessert jelly Delecta & $4.37^{\mathrm{b}}$ & $3.94^{\mathrm{b}}$ & 2.64 & $2.21^{\mathrm{a}}$ & $2.39^{\mathrm{a}}$ & $2.48^{\mathrm{a}}$ \\
colorless dessert jelly Delecta & 3.76 & 3.77 & 2.87 & 2.52 & 2.94 & 3.09 \\
energy gel cola Concap & 0.11 & 0.09 & 0.15 & 0.16 & 0.15 & 0.20 \\
\hline
\end{tabular}

Within rows, different superscript letters indicate significant differences $(\mathrm{P}<0.05)$. 
Table 7. Values of Relative Standard Deviation (\%) of the carrageenan determination.

\begin{tabular}{ccc}
\hline \multirow{2}{*}{ method } & \multicolumn{2}{c}{ sample } \\
\cline { 2 - 3 } & Dr. Oetker & Delecta red \\
\hline spectrophotometry & 3.4 & 2.1 \\
red color photometry & 0.54 & 1.6 \\
photometric titration $(\mathrm{A}=1)$ & 1.6 & 10.3 \\
photometric titration $(\mathrm{A}=1.5)$ & 6.2 & 5.9 \\
photometric titration $(\mathrm{A}=2)$ & 6.6 & 7.7 \\
\hline
\end{tabular}

colorimetry. Although the colour of sample in different images seems to be changed, the contribution of red component is stable (Figure $\mathrm{F}$ in Supplementary material). The highest values of RSD were registered for the titrimetric method. The probable cause is higher degree of sample dilution used in the titrimetric procedure.

\section{Conclusions}

The New Methylene Blue $\mathrm{N}$ dye proved to be well applicable for the rapid determination of carrageenan in jelly deserts. Two methyl groups placed in the aromatic structure facilitate binding the dye with carrageenan macromolecules. Spectral measurements resulted in straight calibration lines. The common drawback of the calibration lines is lack of sensitivity at low carrageenan concentrations (lesser than $7 \mathrm{mgL}^{-1}$ ). The upper limit of determination with the NMB dye depends on type of the carrageenan analysed. Freshly prepared samples should be used in order to ensure good accuracy of the analysis. The spectral measurements should be carried out within 10-15 min after the $\mathrm{NMB}$ dye reagent adding. Smartphone-based colorimetry is also suitable for determining carrageenan. The Red color component is well correlated with the absorbance at $590 \mathrm{~nm}$ and provides straight calibration lines. The procedure of spectrophotometric titration with NMB dye can be recommended for samples with low carrageenan content. This method also leads to straight-line calibration. Smartphone-based colorimetry and spectrophotometry provide quite similar results. Differences were noticed between the results of smartphone-based colorimetry, spectrophotometry and the results of photometric titration $(\mathrm{p}<0.05)$.

\section{Topic relevance}

This manuscript is relevant for food industry and for inspection bodies. The study present new developed methods for carrageenan detection in jellies. The methods were precisely evaluated and compared each other. The spectrophotometric method is well applicable for the rapid determination of carrageenan. The spectrophotometric titration can be recommended for samples with low carrageenan content. Developed Smartphone-based colorimetry is suitable for carrageenan determination, where the red color component correlated with the absorbance at $590 \mathrm{~nm}$.

\section{Acknowledgements}

The work was supported by UVPS Brno project no. FVHE/ Tremlová/ITA2019.

\section{References}

Bednářová, M., Pospiech, M., Jandásek, J., \& Tremlová, B. (2014). Carrageenans in the meat industry: detection using microscopic methods. Maso International: Journal of Food Sience and Technology, $1,11-14$.

Blakemore, W. R., Brant, A. F., Bissland, J. G., \& Bissland, N. D. (2014). Carrageenan analysis. Part 3: Quantification in swine plasma. Food additives \& contaminants. Part A, Chemistry, analysis, control, exposure \& risk assessment, 31(10), 1673-1677. http://dx.doi.org/1 0.1080/19440049.2014.955538. PMid:25164307.

Błaszak, B., Gozdecka, G., \& Shyichuk, A. (2018). Carrageenan as a functional additive in the production of cheese and cheese-like products. Acta Scientiarum Polonorum. Technologia Alimentaria, 17(2), 107-116. http://dx.doi.org/10.17306/J.AFS.2018.0550. PMid:29803212.

Campbell, R., \& Hotchkiss, S. 2017. Carrageenan industry market overview. In A. Q. Hurtado, A. T. Critchley \& I. C. Neish (Eds.), Tropical seaweed farming trends, problems and opportunities: focus on kappaphycus and eucheuma of commerce. Cham, Switzerland: Springer; p. 193-205. http://dx.doi.org/10.1007/978-3-319-634982_13.

Color Picker. (2020). Retrieved from https://imagecolorpicker.com/

Coronel, A., Catalán-Toledo, J., Fernández-Jaramillo, H., Godoy-Martínez, P., Flores, M. E., \& Moreno-Villoslada, I. (2017). Photodynamic action of methylene blue subjected to aromatic-aromatic interactions with poly(sodium 4-styrenesulfonate) in solution and supported in solid, highly porous alginate sponges. Dyes and Pigments, 147, 455-464. http://dx.doi.org/10.1016/j.dyepig.2017.08.042.

Herfurth, J., \& Ulrich, J. (2017). Analysis of hydrocolloids in crystalline material. Chemical Engineering \& Technology, 40(7), 1261-1267. http://dx.doi.org/10.1002/ceat.201600546.

Kilıç, V., Alankus, G., Horzum, N., Mutlu, A. Y., Bayram, A., \& Solmaz, M. E. (2018). Single-image-referenced colorimetric water quality detection using a smartphone. ACS Omega, 3(5), 5531-5536. http:// dx.doi.org/10.1021/acsomega.8b00625. PMid:31458756.

Lakshminarasimhan, N., Sangeetha, D. N., \& Nivetha, G. (2017). Metachromasy of methylene blue due to aggregation over phosphatemodified polymeric carbon nitride. Chemical Physics Letters, 675, 98-103. http://dx.doi.org/10.1016/j.cplett.2017.03.013.

McKim, J. M., Willoughby, J. A. Sr, Blakemore, W. R., \& Weiner, M. L. (2018). Clarifying the confusion between poligeenan, degraded carrageenan, and carrageenan: a review of the chemistry, nomenclature, and in vivo toxicology by the oral route. Critical Reviews in Food Science and Nutrition, 59(19), 3054-3073. PMid:29902080.

Paul, P., Mati, S. S., Bhattacharya, S. C., \& Kumar, G. S. (2017). Spectroscopic, calorimetric, cyclic voltammetric and molecular modeling studies of new methylene blue-polyadenylic acid interaction and comparison to thionine and toluidine blue O: Understanding selfstructure formation by planar dyes. Dyes and Pigments, 136, 205-218. http://dx.doi.org/10.1016/j.dyepig.2016.08.027.

Petrášová, M., Bartlová, M., Pospiech, M., Luňáková, L., \& Tremlová, B. (2016). The use of special PAS Calleja staining for the microscopic detection of selected hydrocolloids in model meat products. Maso Int., 2, 123-130.

Porto, I. S. A., Santos, J. H. No., Santos, L. O., Gomes, A. A., \& Ferreira, S. L. C. (2019). Determination of ascorbic acid in natural fruit juices using digital image colorimetry. Microchemical Journal, 149, 104031. http://dx.doi.org/10.1016/j.microc.2019.104031.

Quemener, B., Marot, C., Mouillet, L., Da Riz, V., \& Diris, J. (2000). Quantitative analysis of hydrocolloids in food systems by 
methanolysis coupled to reverse HPLC. Part 1. Gelling carrageenans. Food Hydrocolloids, 14(1), 9-17. http://dx.doi.org/10.1016/S0268005X(99)00042-9.

Rateni, G., Dario, P., \& Cavallo, F. (2017). Smartphone-based food diagnostic technologies: a review. Sensors (Basel), 17(6), 1453. http:// dx.doi.org/10.3390/s17061453. PMid:28632188.

Serowik, M., Figiel, A., Nejman, M., Pudlo, A., Chorazyk, D., Kopec, W., Krokosz, D., \& Rychlicka Rybska, J. (2018). Drying characteristics and properties of microwave assisted spouted bed dried semirefined carrageenan. Journal of Food Engineering, 221, 20-28. http://dx.doi. org/10.1016/j.jfoodeng.2017.09.023.

Soedjak, H. (1994). Colorimetric determination of carrageenans and other anionic hydrocolloids with methylene blue. Analytical Chemistry, 66(24), 4514-4518. http://dx.doi.org/10.1021/ac00096a018.

Sokolova, E. V., Kuźmich, A. S., Byankina, A. O., \& Yermak, I. M. (2017). Effect of carrageenans alone and in combination with casein or lipopolysaccharide on human epithelial intestinal HT-29 cells. Journal of Biomedical Materials Research. Part A, 05A(10), 2843-2850. http://dx.doi.org/10.1002/jbm.a.36136. PMid:28589676.
Stîngă, G., Băran, A., Iovescu, A., Aricov, L., \& Anghel, D.-F. (2019). Monitoring the confinement of methylene blue in pyrene labeled poly (acrylic acid). Journal of Molecular Liquids, 273, 125-133. http:// dx.doi.org/10.1016/j.molliq.2018.10.023.

Weiner, M. L. (2016). Parameters and pitfalls to consider in the conduct of food additive research, Carrageenan as a case study. Food and Chemical Toxicology, 87, 31-44. http://dx.doi.org/10.1016/j. fct.2015.11.014. PMid:26615870.

Ziółkowska, D., Kaniewska, A., Lamkiewicz, J., \& Shyichuk, A. (2017). Determination of carrageenan by means of photometric titration with Methylene blue and Toluidine blue dyes. Carbohydrate Polymers, 165, 1-6. http://dx.doi.org/10.1016/j.carbpol.2017.02.029. PMid:28363528.

Ziółkowska, D., Shyichuk, A., Lamkiewicz, J., \& Kutsevol, N. (2018). Spectral study on association of thiazine dyes with anionic polymers. Molecular Crystals and Liquid Crystals (Philadelphia, Pa.), 672(1), 133-141. http://dx.doi.org/10.1080/15421406.2018.1542115.

Ziółkowska, D., Syrotynska, I., \& Shyichuk, A. (2014). Quantitation of polyhexamethylene biguanide by photometric titration with Naphthol Blue Black dye. Polimery-W., 59(2), 160-164. http://dx.doi. org/10.14314/polimery.2014.160. 


\section{Supplementary material}

Supplementary material accompanies this paper.

Figure A. Molecular structures of phenothiazine dyes: Methylene Blue (a), Toluidine Blue (b) and New Methylene Blue N (c).

Figure B. Absorbance values at isosbestic points vs. carrageenan concentration and polymer-to-dye molar ratio. The optical path is $10 \mathrm{~mm}$.

Figure C. Vis spectra of reaction mixtures. Concentrations of semi-refined Kerry (a) and iota Eurogum (b) carrageenans are indicated. The optical path is $10 \mathrm{~mm}$.

Figure D. Measuring stand for smartphone-based colorimetry

Figure E. Images of reaction mixtures and corresponding R/G/B values at indicated concentrations of carrageenans.

Figure F. Sample images of reaction mixtures for Delecta red with corresponding R/G/B values.

This material is available as part of the online article from http://www.scielo.br/cta 